\title{
DIRECT MEASUREMENTS OF BETA-STAR IN THE TEVATRON*
}

\author{
M. J. Syphers, Fermilab, Batavia, IL 60510, USA \\ R. Miyamoto, University of Texas, Austin, TX 78712, USA
}

\begin{abstract}
Simultaneous turn-by-turn beam position measurements across collider detector long straight sections provide full phase space information of a free oscillation. For signals with enough coherence (typically 1-2000 revolutions) the amplitude function and its slope at the two BPM's can be directly measured. Results for the Tevatron low- $\beta$ regions with a few percent accuracy are described.
\end{abstract}

\section{INTRODUCTION}

Until recently, values of the amplitude function, $\beta$, through the Interaction Regions of the Tevatron have been inferred using one of two methods: (a) by using event reconstruction to map out the luminous region thus inferring beam emittance and amplitude functions[1], or (b) by performing a series of differential closed orbit measurements while varying steering magnets in the Tevatron, attempt to reproduce the observed orbital deviations with computational models, and inferring $\beta$ at the beam position monitors, from which values of the amplitude function elsewhere are inferred.[2] Both methods require off-line analyses and sometimes many hours of experimental data to obtain a meaningful result. The new Tevatron Beam Position Monitor system,[3] commissioned in 2005, has allowed unprecedented detail of turn-by-turn motion to be measured at the 20-micron level and for thousands of beam revolutions. Such measurements performed with a freely oscillating proton beam excited by a kicker magnet, or with beam excited by an oscillating (AC) dipole field allow for direct measurements of $\beta$ at the BPM locations which is model independent. The improved resolution allows for meaningful measurements to be made with small amplitude beam deflections which are required during high-energy, low-beta operating conditions.

\section{TURN-BY-TURN POSITION MEASUREMENTS}

The position relative to the closed orbit at one BPM, $x$, and the slope of the trajectory relative to the closed orbit, $x^{\prime}=d x / d s$, are related through the amplitude function via $\gamma x^{2}+2 \alpha x x^{\prime}+\beta x^{\prime 2}=a^{2} / \beta$, where $\alpha=-\beta^{\prime} / 2$, $\gamma=\left(1+\alpha^{2}\right) / \beta$, and $a$ is the amplitude of the particle motion as measured at the BPM location. The eccentricity and orientation of the phase space ellipse are given by the values of the amplitude function and its first derivative; the overall size of the ellipse is determined by the amplitude of the particle oscillation.

\footnotetext{
* Work supported by Fermi Research Alliance, LLC under Contract No. DE-AC02-07CH11359 with the United States Department of Energy.
}

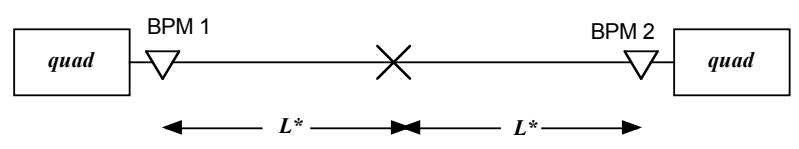

Figure 1: Schematic of long straight section across Interaction Point.

A transverse deflection to the beam will induce a coherent betatron oscillation which can be measured by the BPM's. However, due to inherent non-linearities in the accelerator as well as chromaticity, the phases of the oscillations of individual particles will slip and the signal will "decohere," leading to an apparent damping of the motion. Even though the amplitude of the signal decreases, if it does so slowly enough the data will continue to lie on concentric phase space ellipses and thus can be used in the analysis. Turn-by-turn data out to about one decoherence time can provide many hundreds of data points, increasing the statistics of the measurement. Because of the tune spread which arises due to chromaticity, it is best to measure the amplitude function with "uncoalesced" beam in the Tevatron, for which the momentum spread is many times smaller.

For this discussion we will ignore transverse coupling between the horizontal and vertical motion. The measurement consists of many turns of data obtained simultaneously at two BPM's on either side of the interaction region long straight section, as depicted in Figure 1. At each IR the vertical detectors are separated by $\pm 14.8 \mathrm{~m}$ and the horizontal detectors by $\pm 15.0 \mathrm{~m}$ from the center of the straight section. By taking simple sums of the data from a pair of BPM's the amplitude function and its slope can be determined at each BPM, and the values in between computed.

\section{Moments and End Points}

In one degree of freedom, the transverse motion at a BPM on turn number $n$ is given by $x_{n}=A \sqrt{\beta} \sin (\delta+$ $2 \pi \nu n)$, where $A$ and $\delta$ are determined by the initial conditions of the oscillation due to the applied kick, and $\nu$ is the betatron tune. The slope of the oscillation at the BPM is given by $x_{n}^{\prime}=-\frac{A}{\sqrt{\beta}}[\alpha \cos (\delta+2 \pi \nu n)+$ $\sin (\delta+2 \pi \nu n)]$. Averaging over phases or, equivalently, averaging data over a large number of turns, we find that $\left\langle x^{2}\right\rangle=\frac{1}{2} A^{2} \beta,\left\langle x^{\prime 2}\right\rangle=\frac{1}{2} A^{2} \gamma$, and $\left\langle x x^{\prime}\right\rangle=-\frac{1}{2} A^{2} \alpha$, and so the data can be used to extract the value of $\beta$ and 
its slope $\beta^{\prime}=-2 \alpha$ at the BPM. One can easily verify that $A^{2}=2 \sqrt{\left\langle x^{2}\right\rangle\left\langle x^{\prime 2}\right\rangle-\left\langle x x^{\prime}\right\rangle^{2}}$. The angle $x^{\prime}$ is determined by knowing the distance $2 L^{*}$ between two BPM's and measuring the positions at the two BPM's $x_{1}$ and $x_{2}$ simultaneously, namely $x^{\prime}=\left(x_{2}-x_{1}\right) / 2 L^{*}$, and hence $A^{2} L^{*}=\sqrt{\left\langle x_{1}^{2}\right\rangle\left\langle x_{2}^{2}\right\rangle-\left\langle x_{1} x_{2}\right\rangle^{2}} \equiv \mathcal{A}^{2}$. The final results for $\beta$ and $\alpha$ at the two straight section BPM's are

$$
\begin{gathered}
\beta_{1}=\frac{2\left\langle x_{1}^{2}\right\rangle L^{*}}{\mathcal{A}^{2}}, \quad \beta_{2}=\frac{2\left\langle x_{2}^{2}\right\rangle L^{*}}{\mathcal{A}^{2}}, \\
\alpha_{1}=\frac{\left\langle x_{1}^{2}\right\rangle-\left\langle x_{1} x_{2}\right\rangle}{\mathcal{A}^{2}}, \quad \alpha_{2}=-\frac{\left\langle x_{2}^{2}\right\rangle-\left\langle x_{1} x_{2}\right\rangle}{\mathcal{A}^{2}} .
\end{gathered}
$$

\section{The Amplitude Function Across the IR}

Since we have a drift space between the two BPM's we know that the combination $\left(1+\alpha^{2}\right) / \beta$ is a constant and the amplitude function is parabolic. Then the minimum of $\beta$ and its location relative to the upstream BPM are

$$
\check{\beta}=\frac{2 L^{*}}{\alpha_{1}-\alpha_{2}} \quad \text { at } \quad \check{s}=\frac{2 \alpha_{1} L^{*}}{\alpha_{1}-\alpha_{2}} .
$$

Henceforth, we will refer to the minimum value of $\beta$ as $\beta^{*}$, which may or may not be centered in the straight section. The distance to the minimum from the center of the straight section is $\Delta s^{*}=\check{s}-L^{*}=L^{*}\left(\alpha_{1}+\alpha_{2}\right) /\left(\alpha_{1}-\alpha_{2}\right)$.

\section{A TYPICAL MEASUREMENT}

A typical measurement, Figure 2, shows the first 2000 turns of data from one (upstream) horizontal BPM located at the B0 straight section of the Tevatron, with the mean subtracted. Using this data and the data from the downstream detector, the phase space at each BPM can be reconstructed as shown in Figure 3 for the upstream detector using the first 6000 turns. The ellipse in each figure corresponds to the values of $\beta$ and $\alpha$ obtained directly from the analysis described above. The right portion of this figure shows the same data, but only turns 0-100 (outer points) and turns 6000-6100 (inner points). As can be seen, the orientation of the apparent phase space ellipse does not change over time, even as the particle motion decoheres. Using the phase space data to perform the straightforward

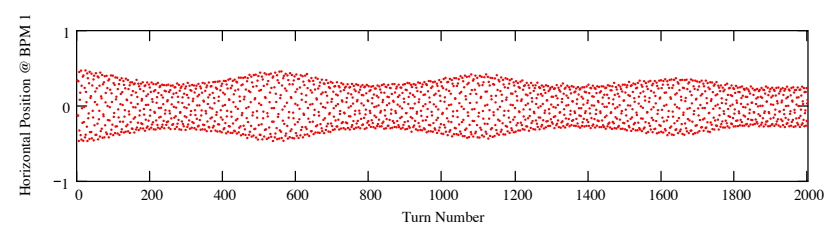

Figure 2: Horizontal BPM data, $x_{1}$, at B0 for a horizontal applied kick at injection energy of $150 \mathrm{GeV}$. The horizontal tune was 20.586. "Beating" due to chromaticity is evident.
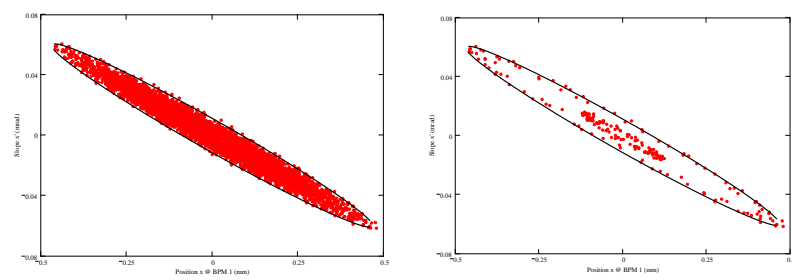

Figure 3: Left: Phase space at the upstream B0 detector. Right: Showing only data for turns $0-100$ and turns $6000-$ 6100. The ellipse in each case corresponds to $\beta_{1}=41.2 \mathrm{~m}$ and $\alpha_{1}=5.26$, a result using the first half of the data (3000 turns) in the analysis.

analysis described above yields values and locations of the minimum amplitude functions through the region.

\section{MEASUREMENTS AT 980 GEV}

The first sets of data at full energy to be analyzed in this way were taken in June 2005 at $980 \mathrm{GeV}$. While the chromatic decoherence was pronounced in the data, it was still viable to use $\sim 1,500$ turns in the analysis. The results of these early measurements are displayed in Table 1 . We see that on this day the values of $\beta_{y}$ are measured with good precision, giving 1-2\% values at each IP. However, the results for $\beta_{x}$ vary with the number of turns used, due to beating in the signal caused by too large a value of chromaticity. Still, the precision was estimated to be less than $10 \%$.

Table 1: Amplitude Functions at $980 \mathrm{GeV}, 2005$ June 23, using 1500 turns.

\begin{tabular}{|c|c|c|c|c|c|}
\hline & & $\beta^{*} / \mathrm{cm}$ & $\delta \beta^{*} / \mathrm{cm}$ & $\Delta s^{*} / \mathrm{cm}$ & $\delta \Delta s^{*} / \mathrm{cm}$ \\
\hline B0 & $\mathrm{H}$ & 34.6 & \pm 0.6 & $+34.2^{\dagger}$ & \pm 0.4 \\
(CDF) & $\mathrm{V}$ & 38.7 & \pm 0.3 & -4.8 & \pm 0.4 \\
\hline \hline D0 & $\mathrm{H}$ & 38.7 & \pm 1.6 & +1.1 & \pm 0.4 \\
& $\mathrm{~V}$ & 40.3 & \pm 0.2 & +2.3 & \pm 0.2 \\
\hline
\end{tabular}

${ }^{\dagger}$ see next Section

\section{NOTES ON MEASUREMENT ERRORS}

The rms readback error of the BPM system is on the order of $20 \mu \mathrm{m}$, and the distances between the BPM centers are known to better than a centimeter, or $<0.1 \%$. The oscillations induced for the measurements above were typically on the order of $1 \mathrm{~mm}$. With these errors alone, one would expect an error on $\beta^{*}$ of better than $2 \%$.

The data indicate that the low-beta optics were tuned close to design and the waists situated within a few centimeters of the middle of the straight section. The exception 
appears to be $\beta_{x}^{*}$ at the CDF detector. Here, the data suggest the waist is off by $40 \mathrm{~cm}$. However, the downstream horizontal BPM at B0 was reading back a closed orbit position of approximately $8 \mathrm{~mm}$ (with maximum excursions, therefore, of about $9 \mathrm{~mm}$ ). All 7 other detectors had the beam centered to about $2 \mathrm{~mm}$ or better. It is known that the response of the BPM is non-linear with position. The scaling of the readback position from the new BPM system is given by $x=26.18 \mathrm{~mm}(A-B) /(A+B)$, where $A$ and $B$ are the induced voltages on the two plates of the pickup. (A survey offset has been ignored.) The sensitivity of the device will be reduced by several percent and the reading will be too low at this size beam offset by as much as $10-20 \%$.[4],[5] If a BPM reading is off by a scale factor $r=1-\delta$, then

$$
d \Delta s^{*} \approx \frac{L^{*}}{2} \cdot \delta, \quad \frac{d \beta^{*}}{\beta^{*}} \approx-\frac{1}{4} \delta^{2}
$$

represent the misinterpretation of the values of $\beta^{*}$ and $\Delta s^{*}$. So, for a downstream BPM at CDF reading back $10 \%$ too low, then this would lead to a misinterpretation of the waist position on the order of $38 \mathrm{~cm}$, while the numerical value of $\beta^{*}$ would only be affected by $-0.25 \%$, as observed.

\section{Solenoid Fields and Beam-Beam Interaction}

While the positions are directly measured, the determination of the slope of the beam trajectory across the straight section has assumed no external fields exist between the two monitors. In reality, each detector has a solenoid field through this region. The characteristic angle, $\theta=B_{s} \ell / B \rho$ will have values $\theta=11-14 \mathrm{mrad}$ at $150 \mathrm{GeV}$ and $\theta=1.7$ $2.2 \mathrm{mrad}$ at $980 \mathrm{GeV}$ for D0 and CDF respectively. Compared to traversal of a pure drift space of length $\ell$, the resulting change in slopes due to the solenoid are $\Delta x^{\prime}=\frac{1}{2} \theta y_{1}^{\prime}$, $\Delta y^{\prime}=-\frac{1}{2} \theta x_{1}^{\prime}$. Using the larger value $\theta=0.0022$ above for $980 \mathrm{GeV}$, we see that for a horizontal kick where the amplitude of the vertical motion is at most $\sim 20 \%$ of the horizontal amplitude (as seen in the data taken at $980 \mathrm{GeV}$ ), then $\Delta x^{\prime} / \hat{x}^{\prime}=\theta\left(\hat{y}^{\prime} / \hat{x}^{\prime}\right) / 2=(0.0022)(1 / 5) / 2=0.02 \%$ on a per turn basis.

One major difference between the measurements discussed here (as well as differential orbit measurements) and the deduction of $\beta^{*}$ from the detector luminous region reconstruction is that in the latter the beams are actually colliding. In this case, the beam-beam interaction takes place at each IP, and acts as a focusing lens on each beam. To lowest order, the focal length of the "lens" is given by $1 / f=4 \pi \xi / \beta^{*}$, where $\xi$ is the "beam-beam parameter," $\xi \equiv 3 N r_{0} / 2 \epsilon_{N}, N$ is the number of particles per bunch, $\epsilon_{N}$ is the $95 \%$ normalized emittance, and $r_{0}$ is the classical radius of the proton. For $\xi \sim 0.01$, then $f \sim 3 \mathrm{~m}$.

A gradient error of focal length $f$ introduced where $\beta=\beta_{0}$ produces a perturbation of the amplitude function around the accelerator of amplitude

$$
\left|\frac{\Delta \beta}{\beta}\right| \approx \frac{\beta_{0} / f}{2|\sin 2 \pi \nu|}=\frac{2 \pi \xi}{|\sin 2 \pi \nu|}
$$

and at the location of the error has magnitude $\left(\beta_{0} / f\right) \cot (2 \pi \nu) / 2=-2 \pi \xi \cot (2 \pi \nu)$. So, for $\xi=0.01$, $\Delta \beta^{*} / \beta^{*}=-12 \%$, and the wave around the ring is of amplitude $13 \%$ (using $\nu=20.578$ ). The point is that the measurement conditions are quite different with and without the on-coming beam in the accelerator, and so $\sim 10 \%$ differences between results of the two methods of measuring $\beta^{*}$ should not be too surprising.

\section{USE OF AC DIPOLE}

Because the above analysis is quick and easy to interpret, its use in every day operation is enticing. However, the above assumes a kicked beam measurement, which is destructive in nature. An AC dipole, however, can be used to generate sustained oscillations for many thousands of turns and then the beam restored to its original condition. In this case Eqs. 1-2 give values of $\beta_{d}, \alpha_{d}$ for the driven oscillation and, with knowledge of the tune and modulation frequency, can be used to find $\beta$ and $\alpha$ of the free oscillation. Details of using an AC dipole in this way can be found in [6].

\section{CONCLUSIONS}

Using the newly upgraded Tevatron BPM system, the first direct measurements of the amplitude function across the two interaction region straight sections have been performed. A fast and simple analysis provides determination of $\beta^{*}$ to a few percent accuracy. The use of an $\mathrm{AC}$ dipole opens the door to accurate, quick measurements of $\beta$ around the ring in a non-destructive manner using similar analysis techniques.

\section{REFERENCES}

[1] J. Slaughter, et al., "Tevatron Run II Luminosity, Emittance and Collision Point Size," Proc. 2003 Part. Accel. Conf., 07803-7739-7, 1763-1765 (2003).

[2] V. Lebedev, "Measurement and Correction of Low-Beta Optics At Tevatron," presentation at Fermilab, 2004 July 29, Fermilab internal document, Beams-doc-1313.

[3] S. Wolbers, et al., "Tevatron Beam Position Monitor Upgrade," presented at 2005 Particle Accelerator Conference, Knoxville (May 2005); M. Martens, et al., "Tevatron Beam Position Monitor Upgrade Requirements," Fermilab Report, Beams-doc-554 (2003).

[4] R. Shafer, "Sensitivity of Beam Position Detectors for the Tevatron," Fermilab Report, UPC-101 (May 1979); R. Shafer, R. Webber, T. Nicol, "Fermilab Energy Doubler Beam Position Detector," Proc. 1981 Part. Accel. Conf., IEEE Trans. Nuc. Sci. NS28 (3), 2290-2292 (1981)

[5] R. Miyamoto, et al., "Geometrical Interpretation of Nonlinearities from a Cylindrical Pick-up," these proceedings.

[6] R. Miyamoto, et al., "Tevatron Optics Measurements using an AC Dipole," and "The Tevatron AC Dipole System," these proceedings. 